\title{
Características de familias resilientes frente a los efectos de violencia politica y social en Colombia: San Jose del Playon: Resistir y vivir
}

\section{Characteristics of resilient families against the effects of political and social violence in Colombia: San Jose del Playon: Resist and live}

DOI: http://dx.doi.org/10.17981/cultedusoc.8.2.2017.04

Jorge Ivan Galindo Madero ${ }^{1}$ y Cirit del Carmen Mateus de Oro ${ }^{2}$

\begin{abstract}
Resumen
Los efectos de la violencia política y social en Colombia se evidencian en las voces de las víctimas que han vivenciado sus efectos, la tendencia de las investigaciones ha estado vinculada con la descripción de las huellas traumáticas de las violencias y esto ha permitido visibilizar a las dimensiones del conflicto, el reconocer las herramientas resilientes que los sujetos y familias han desarrollado frente a los eventos traumáticos se convierte en una posibilidad comprender dinámicas que permiten enfrentar estas vivencias. El presente documento describe los resultados de una investigación que caracteriza a familias resilientes frente a la violencia política y social en Colombia, se planteo un estudio de tipo cualitativo con un diseño de estudio de casos, el nivel es descriptivo. Se describe el caso de las familias de la población de San Jose del Playon en el departamento de Bolívar, en medio de un contexto de constante confrontaciones por los parte de los actores armados, se genera un desplazamiento forzado de la población, algunas familias deciden no desplazarse y quedarse en sus casas bajo la sombra de la muerte. Los resultados describen las herramientas resilientes en el contexto de la convivencia con los grupos armados como aquellas acciones que se lograron cuando los grupos violentos abandonaron la zona.
\end{abstract}

Palabras clave: Familias, resiliencia, violencia política, Colombia.

Recibido: Agosto 12 de 2016 / Aceptado: Octubre 30 de 2016.

\footnotetext{
${ }^{1}$ Psicólogo Clínico, Magister en Psicología, Investigador del Subsistema Institucional de Investigación de la Universidad Metropolitana, Subsistema Institucional de Investigación de la Universidad Metropolitana.

${ }^{2}$ Psicóloga, Magíster en Psicoanálisis, Investigador del Subsistema Institucional de Investigación de la Universidad Metropolitana, Subsistema Institucional de Investigación de la Universidad Metropolitana.
} 


\begin{abstract}
The effects of political and social violence in Colombia are evident in the voices of the victims who have experienced its effects, the trend of the investigations has been linked to the description of the traumatic traces of violence and this has made it possible to visualize the dimensions of the conflict, recognizing the resilient tools that subjects and families have developed in the face of traumatic events, it becomes a possibility to understand dynamics that allow us to face these experiences. This document describes the results of an investigation that characterizes resilient families in the face of political and social violence in Colombia, a qualitative study was proposed with a case study design, the level is descriptive. The case of the families of the population of San Jose del Playon in the department of Bolívar is described, in the middle of a context of constant confrontations by the armed actors, a forced displacement of the population is generated, some families decide not to move and stay in their homes in the shadow of death. The results describe the resilient tools in the context of coexistence with armed groups as those actions that were achieved when violent groups left the area.
\end{abstract}

Keywords: Families, resilience, political violence, Colombia.

\title{
Introducción
}

En Colombia el conflicto armado tiene un origen complejo, este se puede plantear desde la problemática ligada a la tenencia de la tierra en tanto grandes extensiones de territorio fértil del país pertenece a una pequeña proporción de la población Colombiana; Un problema ligado a la participación política en la que grupos políticos no se sienten representados y son amenazados por sus ideologías y la poca presencia y acompañamiento que históricamente el estado Colombiano ha realizado a diversos sectores campesinos del país.

El mapa del conflicto se complejiza en tanto los actores armados encuentran apoyo y financiación de carteles del narcotráfico y sectores tanto civiles como políticos que encuentran en guerrillas o paramilitares un brazo armado para defender sus intereses.

Es la población civil la que queda en medio de los actores armados, en especial la campesina ya que es en pequeñas poblaciones y en sectores campesinos donde se ha desarrollado en forma más extensiva las diversas formas de la violencia (Duarte, 2014)

De esta forma la población civil ha establecido diferentes formas de relación con los grupos armados, la territorialidad es uno de los aspectos básicos para comprender el sentido que pueden tener las relaciones entre la población civil y los grupos armados. El territorio se convierte en lugar de transito de tropas, armas y material relacionado con actividades del narcotráfico, el control del territorio y la población civil se convierte en un trofeo de guerra y permite asegurar empoderamiento militar, económico y político en la zona.

Los grupos guerrilleros tienden a establecer diversos grados de permanencia territorial, de esta forma se pueden presentar incursión en donde se genera una fuerte influencia en la población civil, de permanencia rotativa en la que el grupo transita periódicamente por 3 o 4 municipios generando influencia en la población civil y el uso de territorio de tránsito en que utiliza la zona como un territorio de movilidad. (Leon, 2016) 
Los grupos paramilitares están enfocados en erradicar de la zona a aquellos miembros de la población civil que pueden ser considerados como colaboradores o pertenecientes a grupos de guerrilla, sus actos son vinculados con masacres y generan desplazamiento masivo de la población civil. Logran tomar control de la cabecera municipal en forma permanente y modifican el mapa político de la región.

Cuando el grupo armado toma control permanente en una zona, se generan formas de relación con la población civil que han sido nombradas como anclaje endógeno, aquí los grupos armados logran control sobre la población civil, generando funciones de regulación de la vida de las comunidades, se generan restricciones en a la libertad de la comunidad, violación de derechos fundamentales.

De los relatos de las vivencias del conflicto, las voces de aquellos que no se desplazan sino que por el contrario quedan en sus hogares, en medo de poblaciones desocupadas y con viviendo con los actores armados señala una forma de vivir en medio del conflicto, esta se puede nombrar como una posición de resistencia civil frente a la presencia de grupos armados en una comunidad.

Los relatos sobre los efectos psicosociales y psicológicos de las víctimas del conflicto armado han estado en la mayoría de los casos a reconocer los efectos psicopatológicos y psicosociales que genera la violencia, además de esto, el conflicto a develado como en algunos sujetos, familias y comunidades surgen respuestas que implican una respuesta de transformación positiva frente a la adversidad de la violencia. Estos sujetos no se quedan anclados en el dolor y reconstruyen sus vidas en forma positiva, el reconocimiento de las herramientas con las cuentan las personas para afrontar vivencias de estrés o traumáticas hace parte de los estudios sobre resiliencia.

La resiliencia se puede definir como la capacidad del ser humano para hacer frente a las adversidades de la vida, superarlas e inclusive, ser transformados por ellas (Grotber, 1995), es importante para el estudio de la resiliencia comprender el proceso de enfrentamiento que se presenta frente a las adversidades, las forma como se dinamiza la superación de las dificultades y la transformación que se genera en la construcción de respuestas resilientes. (Andrade, 2011).

En medio del dolor de la tragedia del desplazamiento se puede ubicar en las voces y vivencias de las víctimas del conflicto, experiencias de familias que luego de vivenciar los avatares de la violencia, ha logrado generar respuestas resilientes frente al desplazamiento y sus efectos desestabilizadores, el concepto de resiliencia se convierte en un punto de anclaje clave para comprender estas experiencias.

Los primeros estudios internacionales sobre la resiliencia estaban enfocados en niños y niñas, quienes se desarrollaban en contextos de vulnerabilidad psicosocial y aun así contradecían las exceptivas de presentar trastornos mentales pese variable adversas que se presentaban en su entorno (Masten, 2001) las primeras hipótesis sobre la resiliencia la vinculaban a invulnerabilidad (Rutter, 1993) luego se reconoce que en la resiliencia responde a factores de tipo subjetivo y por consiguiente puede promoverse (Infante, 2005)

Las conceptualizaciones sobre la resiliencia se pueden agrupar en cuatro grandes bloques (Garcia, 2013): 
Las definiciones que apuntan a la comprensión de la resiliencia desde la adaptabilidad del sujeto a las circunstancias vivenciadas en el pasado y el presente (Wenner, 1992); las definiciones de resiliencia que están enfocadas a las capacidades o habilidades de las personas (Grotberg, 1995); aquellas que hacen énfasis en los factores internos y externos que propician la resiliencia (Vanistendael, 2003) y las definiciones de resiliencia que apunta tanto a la adaptación como al proceso mismo (Luthar, 2006).

Es de esta forma que no existe una única definición de resiliencia y esta puede tomar diversos matices de acuerdo a los autores: Se señalan definiciones que resaltan sus aspectos biológicos: La resiliencia es un proceso dinámico, constructivo, de origen interactivo, sociocultural que conduce a la optimización de los recursos humanos y permite sobreponerse a las situaciones adversas. Se manifiesta en distintos niveles del desarrollo, biológico, neurofisiológico y endocrino en respuesta a los estímulos ambientales. (Kotliarenco, 2011); otras definiciones resaltan las capacidades individuales para resolver situaciones que requieren respuesta con entereza: La resiliencia es la capacidad que posee un individuo frente a las adversidades, para mantenerse en pie de lucha, con dosis de perseverancia, tenacidad, actitud positiva y acciones, que permiten avanzar en contra de la corriente y superarlas. (Yturralde, 2006). Uno de los autores que presenta mayor citación en el momento de definir este concepto es Grotber, el define la resiliencia como la capacidad del ser humano para hacer frente a las adversidades de la vida, superarlas e inclusive, ser transformados por ellas, entre sus propuestas teóricas se resalta el trabajo que realiza para comprender la resiliencia tanto en niño y como también en adolescentes (Grotberg, 1995)

Existen diversos autores que categorizado o delimitado a las herramientas de personas resilientes, un ejemplo de esto se encuentra en Fernández-Abascal (FernándezAbascal, 2009) quien señala: Sentido de la autoestima fuerte y flexible, Independencia de pensamiento y de acción, Habilidad para dar y recibir en las relaciones con los demás, Alto grado de disciplina y de sentido de la responsabilidad. Reconocimiento y desarrollo de sus propias capacidades, Una mente abierta y receptiva a nuevas idea, disposición para soñar, Gran variedad de intereses. Un refinado sentido del humor, La percepción de sus propios sentimientos y de los sentimientos de los demás, Capacidad para comunicar estos sentimientos y de manera adecuada, Una gran tolerancia al sufrimiento, Capacidad de concentración, Las experiencias personales son interpretadas con un sentido de esperanza, Capacidad de afrontamiento, Apoyo social

El estudio de la resiliencia implica el reconocimiento de los factores de riesgo; las condiciones de vulnerabilidad y los mecanismos de protección que presentan las personas o grupos que desarrollan respuestas resilientes.

Cuando se reconocen los recursos que tienen las familias para superar eventos como el desplazamiento forzado, nos remitimos al concepto de resiliencia familiar. (Walsh, 2005). Este abordaje de la forma como los individuos y familias afrontan los efectos de la violencia social, responde comprender el fenómeno desde las habilidades y destrezas que tienen los sujetos y no solo desde su déficit y coloca como meta la promoción de estas dinámicas en aquellos que han sido afectados por este tipo de eventos. En esta mirada es importante el reconocimiento de los factores protectores que presentan las familias frente a eventos estresores o traumáticos (Yunes, 2003). Los estudios en resiliencia familiar estaban asociados a la comprensión de como las familias generan respuestas frente a mo- 
mentos críticos (McCubbin, 2002) y en las herramientas y esfuerzos con los que cuentan para enfrentar la adversidad (Hawley, 1996).

Walsh (Walsh, 2005) señala en el estudio de las familias resilientes la importancia de abordar su sistema de creencias, estos son organizadores del sistema familiar, permiten señalar lugares y límites de los integrantes de la familia, en su construcción interviene el discurso del medio social. El sistema de creencias permite que las familias construyan un sentido a partir de la adversidad e implican las dimensiones de trascendencia y espiritualidad que caracteriza la familia. Un segundo aspecto que señala este autor son los patrones de organización familiar, señalados por los recursos económicos y sociales con los que cuenta la familia, cumplen una importante función en la forma como la familia enfrenta las crisis o experiencias que alteran su funcionamiento. El tercer aspecto es señalado por la comunicación, descrita como la capacidad que tienen los integrantes de la familia en expresar y negociar sus necesidades, Walsh señala como la resolución colaborativa de los problemas, la expresión emocional abierta y la claridad de esta comunicación, son aspectos importantes para su estudio. (Walsh, 2005).

Para que se construya la resiliencia es importante que al menos uno de los adultos logre una re significación de la vivencia en forma positiva, esto luego la movilización a otros miembros de la familia. De igual forma se requiere que surjan algunos acuerdos mínimos en la organización cotidiana de la familia como normas de convivencia, disciplina para los niños, construcción de valores (Walsh, 2004)

En Colombia los estudios sobre resiliencia familiar son incipientes, se resalta el interés de explorar y describir cuales son las herramientas de respuesta resiliente que presentan familias que han sido víctimas de conflicto armado, (Lopez, 2013) esto ha implicado una forma diferente de comprender las dinámicas de las víctimas, sin negar su dolor al tiempo que se resalta la capacidad que tiene para ser más allá de la adversidad. Las familias que construyen respuestas resilientes frente al desplazamiento forzado por la violencia, ha sido tema de interés de diversos investigadores, entre los hallazgos de estos estudios se encuentra:

Las más importantes movilizaciones en el sistema familiar se presentan en los primeros meses luego del desplazamiento (Gonzalez, 2004), el soporte económico es una prioridades en este momento (Lopez, 2013) En relación a como se presenta estos cambios sobresale el hecho de que las personas más jóvenes deben separarse del sistema familiar para poder conseguir un trabajo que de sustento económico a la familia; en ocasiones miembros de dos familias se unen circunstancialmente como una forma de responder a las adversidades; (Gonzalez, 2004); se presenta una crisis de roles debido a que las mujeres encuentran mayores posibilidades laborales que los hombres, esto genera crisis en el sistema en tanto el hombre es considerado tradicionalmente como proveedor, en la medida que el hombre avanza en edad se disminuyen sus posibilidades laborales afectando en diferentes esferas de si vida, (Meertens, 1999) esta crisis puede conllevar a que incluso el padre abandone el hogar. (Gonzalez, 2004).

El desplazamiento forzado no solo implica la movilización geográfica de la familia, sino la perdida de la territorialidad, la desorganización de la cohesión familiar, alteración en los procesos de socialización, la separación de los miembros de la familia, crisis en el sistema de pareja, traumatismos importantes generados por la alteración de las roles familiares. (Palacion, 1999) 
En las familias que logran respuestas resilientes frente al desplazamiento forzado, comprende este como un evento externo que las une, que permite el fortalecimiento de lazos familiares, las acciones están dirigidas a la encontrar el apoyo mutuo para enfrentar situaciones de estrés (López L.2007). La capacidad para hacer cambios y responder a la adversidad desde un sistema familiar flexible es una de los recursos que propicia la resiliencia familiar, las familias resilientes logran superar la dinámica responder solo a las necesidades inmediatas y desarrollan habilidades para la planeación, el esforzarse por cumplir metas, el reconocimiento de aquello que es prioritario (Gonzalez, 2004). El reconocimiento y vinculación a las redes de apoyo se convierte en característica de las familias resilientes en tanto esto les permite relacionarse en forma positiva con la comunidad receptora y encontrar nuevas posibilidades laborales y de educación (Gonzalez, 2004)

Mientras la mayoría de los estudios han estado centrados en familias que vivencian el desplazamiento, la descripción de familias que logran respuestas resilientes en el marco de la convivencia continua con grupos armados en Colombia, es un tema que requiere de una mayor exploración.

La experiencia de las familias de la población de San José del Playón en el departamento de bolívar, responden a estas característica, este es de los corregimientos del municipio de María la Baja ubicado en el departamento de Bolívar es San Jose del Playon, un pueblo que históricamente ha estado condenado al olvido de los líderes políticos de la región, con dificultades en el suministro de los servicios públicos, en especial el agua potable ya que no cuenta con alcantarillado, aislado de la cabecera municipal debido a que no contaba con una carretera pavimentada. (Universal, 2014)

En contraposición a las precarias condiciones de calidad de vida de sus habitantes, San Jose del Playon ha sido punto de interés de diferentes grupos armados debido a que tiene una ubicación estratégica que ha sido utilizada para el tránsito de tropas de las FARC, el ELN, el EPL y grupos paramilitares (mujeres, 2013) .

Esta población se encuentra en medio de la montaña y el camino hacia el mar, es por esto que los grupos utilizaban su territorio para transportar tropas, alimentos e incluso secuestrados. Esto colocaba a sus habitantes en la mira de los armados e incluso del ejército nacional que encontraba en ellos unos posibles colaboradores de grupos guerrilleros.

Aquí hubo varios grupos. Bueno, desde allí empezamos a sufrir porque siempre paraban esos grupos por allí. Entonces el ejército se metía y quería culpar a los campesinos, porque decían que nosotros compartíamos con ellos. Pero la verdad es que tristemente éramos unos campesinos y no teníamos que ver con el que pasara o dejara de pasar por allí. (mujeres, 2013)

Cada grupo armado que tomo control de zona tenía su propia forma de promover el terror en sus habitantes, en los tiempos del EPL se presentaron asesinatos, luego con la FARC se recuerdan secuestros realizados en la población civil. Para el año 1999 hacen presencia los paramilitares quienes perpetran masacres en la zona. 
La guerra aquí la estamos viviendo hacen 26 años. Empezamos con la guerrilla, primero con un grupo que se llamó el ELN. Después el ELN se retiró y llegó las FARC. Desde allí empezamos a sufrir y con todo nos quedamos allá en el monte, pasando muchas luchas. Ya en 1999 empezaron los paramilitares a hacer masacres, no en la misma vereda donde nosotros vivíamos, pero sí muy cercano. Entonces en el 2000 nos tocó desplazarnos hacia acá y siguieron sucediendo tantos casos acá que salimos de un mal prácticamente para otro, porque acá también eran las cosas peores. (mujeres, 2013)

La lucha por el territorio por parte de los grupos armados propicio que se realizara un desplazamiento masivo de sus habitantes a finales de la década de los 90s, por esto el pueblo quedo solo y abandonado, los que permanecían en el pueblo representaba menos del 10\% del total de la población

Los habitantes que no se desplazaron sufrieron las formas de vida que imponían los paramilitares implicaba: Retenes en los puntos de comunicación del corregimiento para controlar la entrada y salida de civiles del casco urbano; Control sobre el acceso a los alimentos por parte de la población civil; Control y castigo a quienes generen conflictos entre vecinos; Castigos por robos que podía implicar la pena de muestre.

Los motivos por los que estas familias no se desplazan son variados, incluyen: El deseo de no perder sus casas y sus tierras, el generar una respuesta distinta frente a la violencia de los grupos armados o el temor a la incertidumbre del desplazamiento.

Durante el tiempo que se presentó la convivencia con grupos paramilitares, se generaron diversas acciones que se convierten en violación de los derechos humanos y del derecho humanitario internacional: Abuso sexual, asesinato, desaparición forzada, torturas y secuestro.

La situación de constante zozobra que viven los habitantes de San José del Playón conllevo a alteraciones en la salud de muchos de los habitantes: Hipertencion asociada a factores emocionales, síntomas de ataques de pánico y de trastorno por estrés post traumático, trastornos del sueño y fobias específicas.

Yo no sé cómo sobrevivimos, porque no había donde comprar nada. Ni podíamos salir porque teníamos mucho miedo, porque era terrible. Quedar en que no había nadie, sino perros y miedo. Toda la gente tenía miedo. La gente no dormía. Playón está compuesto por puros hipertensos. Aquí la gente está joven y ya es hipertensa. Fue tanto susto, tanta retención que el miedo le hizo a la gente, que hoy estemos casi todos enfermos de lo mismo.

Dolor de cabeza. Temor a tanta soledad que se vivió aquí y tanta violencia y he quedado con miedo a toda persona extraña. Yo cuando veo una persona extraña ya estoy siendo cobarde, con temor. Hasta este momento yo les tengo miedo a todas las personas que portan armas. Es muy difícil hablar con un soldado. Yo puedo hablar con él pero siento ese dolor. Toda persona con arma, sea soldado, sea policía, le tengo miedo. ( Mujeres, 2013)

Los impactos de la violencia también se expresaron en los niños, niñas y adolescentes que vivieron estas circunstancias, los cuales pueden ser entendidos desde la salud hasta alteraciones en tránsito por la escuela ya que durante los años que los paramitares hicieron presencia muchos aspectos de la vida cotidiana de la población se vio alterado incluyendo la educación. 


\section{Metodología}

La investigación responde diseño narrativo, este permite comprender una sucesión de eventos a través de historias o narrativas de quienes, siguiendo una línea de tiempo hasta el presente (Sampieri, 2015) En este caso se describen las características y dinámicas que se presentaron y presentan en sistemas familiares que responden en forma resilientes frente al desplazamiento forzoso. El estado de la investigación es descriptivo. La temporalidad de la información se vislumbra en dos direcciones: Retrospectiva en tanto interesa reconocer en el pasado las vivencias durante y luego del desplazamiento que permiten comprender de qué forma se construyó la respuesta resilientes en la familia y prospectiva ya que se valora las vivencias relacionadas con el presente del sistema familiar

El diseño de la investigación esta direccionada por el modelo de estudio de casos en el cual cada familia es tomada como un caso, el cual responde a las exigencias que implica nombrar a una

Los textos retomados se para el presente texto responden a historias relatadas en su mayoría desde voces de mujeres, ellas se convierten en el pilar de muchas familias resilientes frente a la violencia del conflicto armado, la reflexión de sus historias genera aprendizajes a todos aquellos profesionales y equipos sociales que promueven intervenciones tanto en las familias como en comunidades afectadas.

La mujer cumple un papel esencial en la mayoría de los casos como pilar de base para el sostenimiento de la familia, por el lugar protagónico que puede tener en el sostenimiento familiar cuando el hombre muere o es desaparecido en medio del conflicto armado. Se tomaron cinco casos de estudio ubicados geográficamente de la siguiente forma:

El análisis de los datos parte de unas categorías base que luego unen con las generadas por el proceso de recolección de información, de esto forma se logra una categorización final. En este proceso se analiza comparativamente las narrativas de los participantes que permitan identificar unidades, generar categorías, valorar los contextos en los cuales se logra la respuesta resiliente y las consideraciones comunes a cada familia que han permitido la resiliencia.

\begin{tabular}{|c|c|}
\hline Nombre de la categoría & Descripción de la categoría \\
\hline $\begin{array}{l}\text { Características del } \\
\text { sistema familiar }\end{array}$ & $\begin{array}{l}\text { Describe las características de la familia desde las teorizaciones sobre } \\
\text { tipos y modelos de familia. }\end{array}$ \\
\hline $\begin{array}{l}\text { Respuestas frente al } \\
\text { desplazamiento forzado }\end{array}$ & $\begin{array}{l}\text { Describe la forma como la familia asume el proceso de desplazamiento } \\
\text { forzado, desde los impactos que este genera en: movilizaciones en el } \\
\text { sistema familiar, roll de hombre, de la mujer, de los hijos; relación del } \\
\text { sistema familiar con otros sistemas familiares y contextos comunitarios, } \\
\text { espacios para la re significación de la vivencia }\end{array}$ \\
\hline $\begin{array}{l}\text { Efectos de las acciones } \\
\text { resilientes en las familias }\end{array}$ & $\begin{array}{l}\text { Describe los efectos de las acciones resilientes en el sistema familiar, } \\
\text { en la forma como se vincula con otros sistemas de familias y contextos } \\
\text { comunitarios. }\end{array}$ \\
\hline
\end{tabular}




\section{Resultados}

\begin{tabular}{|c|c|}
\hline $\begin{array}{l}\text { Respuestas resilientes } \\
\text { (microsistema) }\end{array}$ & Efectos de las respuestas resilientes \\
\hline $\begin{array}{l}\text { Re organización de } \\
\text { la familia frente a la } \\
\text { respuesta de necesidades } \\
\text { primarias }\end{array}$ & $\begin{array}{l}\text { Movilización de los jóvenes para buscar trabajo } \\
\text { Familias } \\
\text { La mujer busca posibilidades laborales } \\
\text { Familias circunstancialmente constituida }\end{array}$ \\
\hline $\begin{array}{l}\text { Replanteamiento de los } \\
\text { lugares de genero }\end{array}$ & $\begin{array}{l}\text { Replanteamiento de discursos machistas y patriarcales } \\
\text { Empoderamiento de la mujer en la familia y la comunidad } \\
\text { La madre presenta multi funcionalidad }\end{array}$ \\
\hline $\begin{array}{l}\text { Herramientas para la } \\
\text { resolución de conflictos }\end{array}$ & $\begin{array}{l}\text { Fortalecimiento de la comunicación } \\
\text { Formas democráticas y horizontales para la resolución de conflictos }\end{array}$ \\
\hline $\begin{array}{l}\text { Apoyo de la familia } \\
\text { extensa y extensiva }\end{array}$ & $\begin{array}{l}\text { Fortalecimiento de las relaciones con la familia extensa y extensiva } \\
\text { Crisis frente a las movilizaciones que genera la presencia de miembros de la } \\
\text { familia extensa y extensiva }\end{array}$ \\
\hline $\begin{array}{l}\text { Protección a niños y } \\
\text { niñas }\end{array}$ & $\begin{array}{l}\text { Los niños y niñas cuentan con espacios para el desarrollo de la lúdica en } \\
\text { contextos de cuidado y protección } \\
\text { Necesidad de que los niños y niñas entre al ámbito escolar }\end{array}$ \\
\hline $\begin{array}{l}\text { Familias } \\
\text { circunstancialmente } \\
\text { constituidas }\end{array}$ & $\begin{array}{l}\text { Se crean ollas comunitarias para responder a las necesidades alimenticias de } \\
\text { las familia } \\
\text { Se comparte techo para responder a las necesidades de vivienda } \\
\text { Se generan conflictos en la búsqueda de normas de convivencia entre las familias } \\
\text { Perdida de intimidad de las diferentes familias en convivencia }\end{array}$ \\
\hline Educación & $\begin{array}{l}\text { La culminación de estudios permite una mejor ubicación laboral a los miembros } \\
\text { de la familia } \\
\text { Los miembros de la familia ganan un discurso sobre derechos humanos, derecho } \\
\text { humanitario internacional y derecho de las victimas }\end{array}$ \\
\hline $\begin{array}{l}\text { Apoyo comunitario y } \\
\text { gubernamental frente a } \\
\text { necesidades básicas }\end{array}$ & $\begin{array}{l}\text { Fortalecimiento de la relación de la familia con las entidades gubernamentales } \\
\text { Fortalecimiento de la relaciones de las familias con comunidades religiosas } \\
\text { Creación de fundaciones y otras entidades por mienbrois de comunidad con el } \\
\text { objetivo de trabajar por las necesidades comunitarias }\end{array}$ \\
\hline $\begin{array}{l}\text { Espacios comunitarios } \\
\text { para la re significación } \\
\text { de las experiencias } \\
\text { vinculadas con el } \\
\text { desplazamiento }\end{array}$ & $\begin{array}{l}\text { Se logra la re significación de experiencias } \\
\text { Empoderamiento de la mujer en la familia y la comunidad } \\
\text { Visualización de la comunidad en el contexto regional y nacional }\end{array}$ \\
\hline
\end{tabular}

\section{Características del sistema familiar}

Las familias de San Jose del Playon se caracterizaban por sistemas nucleares donde exista una distribución de labores por género y edad, los hombres se dedicaban a las labores del campo y el comercio, mientras las mujeres estaban en casa atendiendo actividades domésticas y cuidando de los niños y niñas, los adolescentes y jóvenes ayudaban a sus padres en labores del campo.

Sistema familiar nuclear antes del desplazamiento cuenta con el apoyo de la familia extensa en acciones que permiten responder a obligaciones laborales del campo, se describen con herramientas que les permite responder a las crisis evolutivas del sistema familiar, de igual forma la familia extensiva cumple funciones de acompañamiento en labores domésticas y responsabilidades laborales. 


\section{Respuestas resilientes como afrontamiento de la violencia política}

\section{- Re organización de la familia frente a la respuesta de necesidades primarias}

Luego de la convivencia continua con grupos armados se presentan organizaciones familiares de tipo mono parental debido a la muerte o desaparición de hombres., el sistema familiar es abierto a construir vínculos con otras familias como una forma de responder a las necesidades de vivienda, alimentación y protección de niños, niñas y ancianos

Movilización de los jóvenes para buscar trabajo. Los jóvenes deben desplazarse de la población debido a que se convierten en objetivo militar de los grupos armados debido a su posible relación con grupos guerrilleros, esto implica que deben buscar trabajo y vivienda en otras poblaciones en las cuales no exista influencia de grupos paramilitares.

La mujer busca posibilidades laborales. Se evidencia cambios en la forma como se comprende los lugares y espacios laborales frente al género, debido a la ausencia de hombres en los hogares por la violencia o la dificultad para conseguir trabajo, la mujer asume un lugar protagónico en economía familiar, busca trabajo para lograr el sostenimiento familiar.

Familias circunstancialmente constituida. Organización de familias para responder a las circunstancia bajo el principio de la solidaridad, esto permite que grupos que familias se organicen para responder a las necesidades de los niños, niños, adolescentes $\mathrm{y}$ ancianos.

La solidaridad entre las familias permite responder a necesidades básicas como protección frente a los violentos, varias familias viven en una casa para protegerse, alimentación con la realización de ollas comunitarias.

Esto genera como efecto la creación de ollas comunitarias para responder a las necesidades alimenticias de las familias; frente a la vivienda se comparte el techo, todo esto viene acompañado de conflictos generados por la pérdida de la intimidad y la necesidad de crear normas de convivencia entre las familias

\section{- Replanteamiento de los lugares de genero}

Esto implica el replanteamiento de discursos machistas y patriarcales, un empoderamiento de la mujer en la familia y la comunidad y la multifuncionalidad de la mujer

\section{Respuestas frente al afrontamiento de la violencia:}

Lo anterior generan el empoderamiento de la mujer en marco familiar y comunitario, ahora ellas reconocen habilidades y destrezas para responder de diferente forma las responsabilidades del hogar.

- Realización de actividades en conjunto por parte de varias familias como una forma de protección: El pescar, realizar labores de agricultura y ganadería se realizan en conjunto.

- Organización de actividades recreativas en las que participan miembros de varias familias, aquí el jugar futbol en los adultos incluyendo a las mujeres, la danza y la lúdica en los niños, niñas y adolescentes se convirtió en una forma de afrontamiento. 


\section{Efectos de las acciones resilientes en las familias:}

Luego de la retirada de los grupos armados de la población se presenta la búsqueda de apoyo en el estado, grupos religiosos y entidades encargadas de la atención a la víctima, integrantes de familias asumen un roll de liderazgo frente a la comunidad y sus necesidades. Se generan espacios en las familias y en comunidad para expresar emociones y re significar vivencias relacionadas con la vivencia de las violencias del conflicto armado.

- Empoderamiento de la mujer dentro del núcleo familiar y en el contexto comunitario, constitución de una comunidad en la que varias familias comparten obligaciones de alimentación y vivienda., relaciones sostenibles entre las familias y el estado, fundaciones y grupos religiosos para encontrar respuesta a las necesidades de la comunidad.

Cuando las condiciones lo permitieron varias adultos le apostaron a la educación, unas terminaron el bachillerato y otras fueron accediendo a estudios técnicos y tecnológicos,

Yo personalmente, después que eso pasó, me capacité. Yo apenas llegué hasta la primaria, terminé mi bachillerato. Me capacité como madre comunitaria y hasta el momento estoy ejerciendo el cargo de madre comunitaria y me ha ayudado mucho. También empezamos a estudiar. Validábamos bachillerato, ya eso fue en el 2003 para acá. Empezamos a estudiar los sábados. Al grupo del 2003 nos tocó en San Pablo. Como cuarenta nos graduamos. (mujeres, 2013)

La educación le permitió a muchos de sus habitantes reconocer cuáles son sus derechos, como habían sido vulnerados y a forma de buscar una restitución de los mismos, mejoraron las condiciones económicas de la población y la región ya que se introdujeron nuevas formas de tratamiento de la tierra y de sus riquezas, la población se fortaleció en el cultivo de la yuca y el ñame, se logró el apoyo del Plan Mundial de Alimentos para generar nuevas posibilidades de sustento y crecimiento económico de la población

Con el tiempo se inicia capacitación en la elaboración de proyectos sociales y se unen miembros de la comunidad para presentar iniciativas al gobierno nacional y entidades de apoyo internacional que permitan el progreso de la población. En el año 2003 se constituye la Corporación San José del Playón con la se incentivan proyectos que propician acciones para el presente y futuro de la comunidad.

- La creación de comedores para los niños y niñas de la comunidad, llegando a recibir 100 infantes diariamente, quienes presentaban problemas de desnutrición.

- Talleres lúdicos par niños y niñas víctimas del conflicto armado dictados por psicológicos dirigidos

- Apoyo psicológico para la comunidad afectada por los hechos violentos

- Atención a comunidad desplazada no solo del corregimiento si no de otros sectores aledaños que lo requieran

- Cursos y programas comunitarios en la temática de derechos humanos y derecho humanitario. 


\section{Referencias}

Andrade, M. L. (2011). Resiliencia Familiar: Nuevas perspectivas en la promocion y prevencion en salud. Diversitas: Perspectivas en Psicologia , 43-55.

Aristizabal, A. C. (2013). Como nos empezo la violencia . Contexto, Uiversidad Pontifiicia Bolivariana, 3.

Bello, M. (2003). El desplazamiento forzado en Colombia: Acumulacion de capital y exclusion social. Revista aportes Andinos,Globalizacion, migracion y derechos humanos .

Gomez, E. (2010). Resiliencia Familiar un enfoque de investigacion e intervencion con familias multiproblemicas . revista de Psicologia , 103- 126.

Gonzalez, C. (2004). Transformacion y resiliencia en familias desplazadas por la violencia en Bogota. Revista de estudios sociales, 223- 230.

Grotber, E. (1995). Una guia para la promocion de la resiliencia en niños. The Bernard van Leer Fundation .

Hawley. (1996). Toward a definition of family resilience: integrating life span and family perspectives. Family process, 35-23.

Historica, C. N. (2013 ). Informe General del grupo de memoria historica. Basta Yaj. Bogota.

Lopez, L. (2007). Desplazamiento forzado en el oriente Antioqueño, estrategias familiares de sobrevivencia. Medelliin: Comfenalco.

Lopez, O. L. (2013). Efectos y reacciones de las familias frente al desplazamiento forzado en Colombia . En C. O. Laguado, Familia (págs. 265-281). Medellin: Coorporacion de Investigaciones Biologicas.

McCubbin, M. (2002). Family Resiliency in Childhood Cancer. Family Relations, 103111.

Meertens. (1999). Desplazamiento forzado y genero: Trayectorias y estrategias de reconstruccion vital,. Bogota: Universidad Nacional.

Mujeres, R. p. (2013). La verdad de las mujeres en el conflcito armado Colombiano. Ruta pacifica de las mujeres.

Palacion, A. M. (1999). Estres post traumatico y resistencia psicologica en jovenes desplazados. Investigacion y desarrollo, Vol N10.

Roja, C. (2009). Desplazamiento forzado en conflictos armados: Responder a los desafios . Ginebra: CICR.

Unidas, N. (1998). Principios rectores de los desplazamientos Internos . Naciones Unidas.

Universal, E. (25 de 02 de 2014). Habitantes de San Jose del Playon bloquena la via .

Walsh. (2004). Resiliencia familiar. Estrategias para su fortalecimiento. Buenos Aires: Amorrortu editores.

Walsh, F. (1988). El concepto de Resiliencia familiar: crisis y desafío. Sistemasfamiliare, 11 .

Walsh, F. (2005). Fortalecendo a resiliencia Familiar. Sao Paolo : Roca.

Yunes, M. (2003). Psicologia positiva e resiliencia: o foco no individuo e na familia. Psicologia em Estudo, 1- 15. 